\title{
Simultaneous dual-element analyses of refractory metals in naturally occurring matrices using resonance ionization of sputtered atoms
}

\author{
W. F. Calaway \\ Materials Science and Chemical Technology Divisions, Argonne National Laboratory, \\ Argonne, Illinois 60439 \\ R. C. Wiens and D. S. Burnett \\ Division of Geological and Planetary Sciences, California Institute of Technology, \\ Pasadena, California 91125 \\ M. J. Pellin and D. M. Gruen \\ Materials Science and Chemistry Divisions, Argonne National Laboratory, Argonne, Illinois 60439
}

(Received 21 October 1994; accepted 25 January 1995)

\begin{abstract}
The combination of secondary neutral mass spectrometry (SNMS) and resonance ionization spectroscopy (RIS) has been shown to be a powerful tool for the detection of low levels of elemental impurities in solids. Drawbacks of the technique have been the laser-repetition-rate-limited, low duty cycle of the analysis and the fact that RIS schemes are limited to determinations of a single element. These problems have been addressed as part of an ongoing program to explore the usefulness of RIS/SNMS instruments for the analysis of naturally occurring samples. Efficient two-color, two-photon $(1+1)$ resonance ionization schemes were identified for Mo and for four platinum-group elements $(\mathrm{Ru}, \mathrm{Os}, \mathrm{Ir}$, and $\mathrm{Re})$. Careful selection of the ionization schemes allowed Mo or Ru to be measured simultaneously with Re, Os, or Ir, using two tunable dye lasers and an $\mathrm{XeCl}$ excimer laser. Resonance frequencies could be switched easily under computer control, so that all five elements can be rapidly analyzed. In situ measurements of these elements in metal grains from five meteorites were conducted. From the analyses, estimates of the precision and the detection limit of the instrument were made. The trade-off between lower detection limits and rapid multielement RIS analyses is discussed. (C) 1995 American Vacuum Society.
\end{abstract}

\section{INTRODUCTION}

Coupling resonance ionization spectroscopy (RIS) for postionization of sputtered atoms with secondary neutral mass spectrometry (SNMS) is a powerful combination for trace surface analysis. ${ }^{1-4}$ Among the advantages of such an instrument are (1) elemental sensitivities yielding detection limits below one part per billion (ppb), ${ }^{1}$ (2) high selectivity from isobaric interferences, ${ }^{5}$ (3) quantitative measurements relatively devoid of matrix effects, ${ }^{6}$ and (4) high detection efficiencies permitting analyses of small samples with very low material consumption. ${ }^{7,8}$ In the area of planetary materials, the unique capabilities of RIS/SNMS instrumentation are of particular benefit because of the small size of mineral grains that are of interest. In previous investigations, compositional $^{8}$ and isotope ratio ${ }^{7}$ determinations in metals from various meteorites have been reported.

Despite the high useful yield of the RIS/SNMS method, the time required to complete a measurement remains a major concern for these instruments, particularly if the concentration of a number of elements is to be determined. This limitation stems from the repetition rate of high intensity pulsed lasers and the single element nature of RIS. Typically, multielement RIS analyses are performed sequentially; that is, single-element analyses one after the other. Thus, the time required for multielement analyses and the amount of sample consumed increases linearly with the number of elements determined. Clearly, it would be preferable to analyze elements simultaneously by ionizing all species of interest at the same time. This is especially true when sample size limits the amount of material available for an analysis, or when characterization of a material requires knowing the relative abundance of a suite of elements as a function of another parameter such as sample location.

Several techniques for nonselective postionization of secondary neutrals are presently used for multielement analysis. This is the basis of electron impact SNMS instruments ${ }^{9}$ and of instruments that employ multiphoton nonresonance ionization. ${ }^{10}$ While both of these methods achieve the benefits of increased sensitivity and reduced matrix effects that are a consequence of detecting sputtered neutrals, instruments based on these ionization techniques lack the selectivity of RIS/SNMS instruments. Because of this, isobaric interferences often prevent detection of elemental concentrations below $1 \mathrm{ppm}$ for these instruments, particularly in complex matrices such as the meteoritic samples being studied here. In addition, it is more difficult to achieve ionization saturation with these techniques, and this further limits the sensitivity and the ability to perform quantitative measurements. Consequently, there still exists a need to develop methods for multielement analyses that maintain the high selectivity and low sample consumption of RIS/SNMS instruments.

This article demonstrates rapid, multielement analyses with a RIS/SNMS instrument. Our approach employs three features: (1) simultaneous ionization of two elements using RIS, (2) simultaneous measurements of backgrounds, and (3) identifying ionization schemes for a suite of elements within the tuning curve of a single laser. Dual-element analyses 
were conducted using two dye lasers and an excimer laser, reducing the analysis time and sample consumption by a factor of two. The key to this procedure is identifying simple two-color, two-photon ionization schemes that use a common photon for the ionization step (e.g., for promoting an electron from a resonance state to the ionization continuum). Thus, the number of elements that can be detected simultaneously is equal to the number of tunable lasers available for selecting resonance transitions. While this procedure is straightforward, to our knowledge no multielement RIS experiments have previously been reported in the literature. Another consequence of employing a simple two-color ionization scheme is that the same background measurement can be used for all RIS determinations, saving additional time. Selection of ionization schemes that are within the tuning curve of a single laser dye for platinum-group and rare earth elements is relatively easy because of the large number of electronic transitions that they possess.

As a test of these techniques, the concentrations of five refractory elements (Mo, Ru, Ir, Os, and Re) have been measured in metal grains of several ordinary chondrites. The samples were chosen to demonstrate the in situ capability of the RIS/SNMS technique for measuring trace concentrations. In situ RIS measurements for Os in meteoritic samples have been reported in the past. ${ }^{8}$ In addition, the application of RIS/SNMS instrumentation to Re and Os isotope measurements has been reported. ${ }^{11}$ Ion microprobe (SIMS) analyses of Pt-group metals have poor sensitivity and have not been reported. Past measurements using other techniques have mostly been bulk determinations of metal separates, precluding examination of individual grains and the relationship of a grain to surrounding phases. ${ }^{12,13}$ Neutron activation analysis (NAA) has been used to measure trace concentrations in individual grains ${ }^{14}$ and sub-microgram size cosmic dust grains. ${ }^{15}$ However, this technique is incompatible with in situ measurements.

The purpose of this research is twofold: (1) to demonstrate the potential for reducing analysis time and sample consumption during multielement RIS/SNMS determinations by conducting RIS analyses in parallel and (2) to determine in situ trace concentrations of refractory metals in meteoritic samples.

\section{EXPERIMENT}

The RIS/SNMS instrument used for these experiments (SARISA; surface analysis by resonance ionization of sputtered atoms) is a home-built apparatus designed specifically for ultrahigh sensitivity elemental analysis. The instrument is contained in an ultrahigh vacuum chamber $\left(10^{-10}\right.$ Torr) with a load lock system for sample exchange. It consists of an ion source, time-of-flight (TOF) mass spectrometer, and a laser system. The ion source (Colutron G1) is mass filtered and pulsed. For these measurements, the ion gun was operated with argon at $3.5 \mathrm{keV}$ above the target potential and delivered $2 \mu \mathrm{A}$ of current in a $150 \mu \mathrm{m}$ diameter spot. For most experiments, a $2 \mu$ s ion pulse width was used. An energyand angular-refocusing TOF mass spectrometer is employed to perform mass discrimination and noise reduction. ${ }^{1,6,7}$

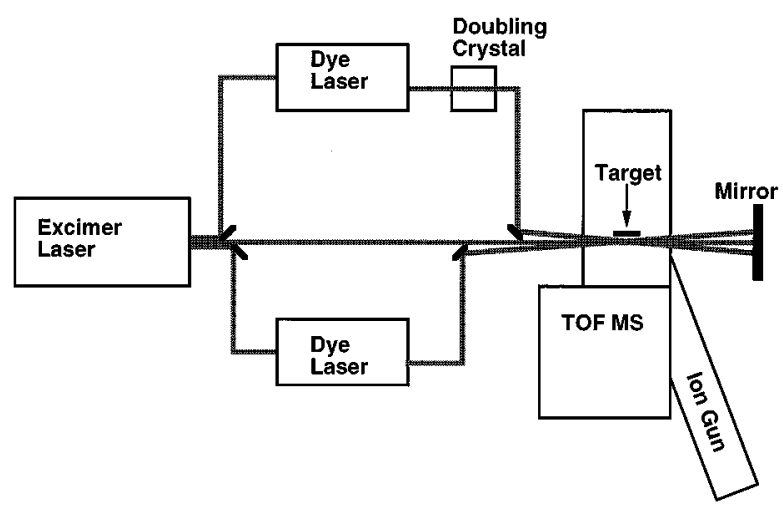

FIG. 1. Schematic drawing of the laser system used for simultaneous dualelement RIS/SNMS measurements.

The laser system consists of two Lumonics HyperDye300 dye lasers pumped by a Lambda EMG-201 excimer laser (Fig. 1). The $\mathrm{XeCl}(308 \mathrm{~nm})$ excimer had an output intensity of $200 \mathrm{~mJ} / \mathrm{pulse}$. The laser beam was split into three pieces in the proportions $80 / 80 / 40$. The two $80 \mathrm{~mJ} /$ pulse beams were used to pump the two dye lasers producing more than 1 $\mathrm{mJ} /$ pulse of tunable light each. The third beam, which was used for the ionization step, was combined with the outputs from the two dye lasers. All three beams were directed into the vacuum chamber, so that they passed through the sputtered flux plume directly in front of the sample target within a volume that could be efficiently extracted into the mass spectrometer. Cylindrical lens telescopes were used where necessary to match the shape of the two dye laser beams in both the vertical and horizontal axes with the extraction volume. Knife-edge slits were used to shape the excimer beam as it entered the vacuum chamber, so that it too is matched in size with the extraction volume. The wavelength selections and scanning of the dye lasers were under computer control. For one of the dye lasers, a $\beta$-barium borate (BBO) crystal was used to frequency double the laser output. The crystal was mounted in an Inrad Autotracker II, so that efficient doubling was maintained when the frequency of the laser was changed.

Shown in Fig. 2 are the ionization schemes used to detect $\mathrm{Ru}, \mathrm{Mo}, \mathrm{Re}, \mathrm{Os}$, and Ir. The criteria employed for the selection of the ionization pathways were (1) the ionization process follows a two-color, two-photon $(1+1)$ scheme with the ionizing photon originating from the $\mathrm{XeCl}$ excimer laser (4 $\mathrm{eV})$, (2) the resonance transition for each element must be sufficiently strong so that saturation is possible by the typical output from a dye laser, and (3) a set of resonance transitions could be found so that only two dyes were needed to detect all five elements. The first criterion is key to the measurements, for it requires that only a single dye laser be used for each element that is to be determined in parallel. The second criterion maximizes sensitivity and minimizes the influence of laser power on signal intensity. Unfortunately, our present experimental arrangement did not achieve total saturation for Os and Ir. The third criterion makes it possible for all five elements to be determined without changing laser dyes.

Detailed spectroscopic data for $\mathrm{Ru}$ and $\mathrm{Mo}$ are 


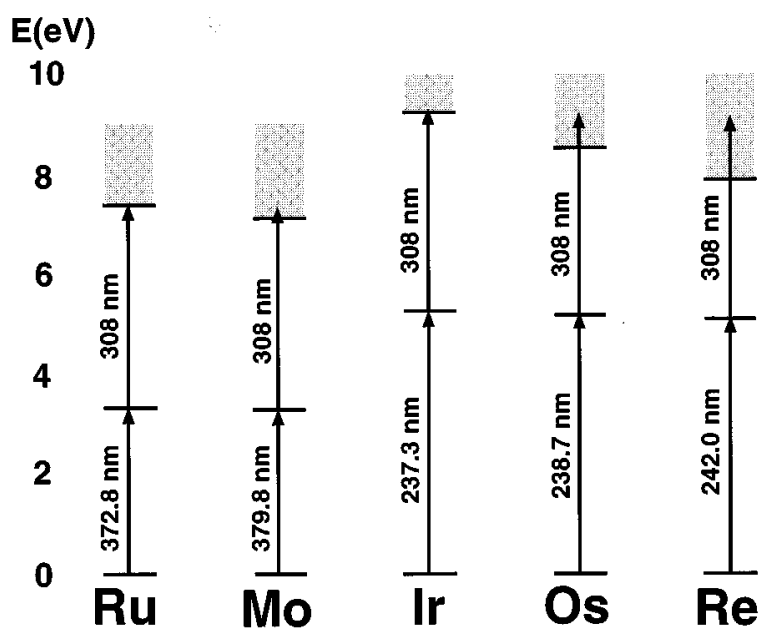

FIG. 2. Partial energy diagrams for $\mathrm{Mo}, \mathrm{Ru}, \mathrm{Re}, \mathrm{Os}$, and Ir showing the resonance ionization schemes that were used for these measurements.

available. ${ }^{16}$ Therefore, it is relatively simple to identify transitions for these two elements that meet the above criteria. The RIS schemes that were used for Ru (Ref. 17) and Mo (Ref. 6) had been tested previously in our laboratory. The $a^{5} F_{5}$ to $z^{5} F_{5}^{0}$ transition for $\mathrm{Ru}(372.8 \mathrm{~nm})$ and the $a^{7} S_{3}$ to $z^{7} P_{4}^{0}$ transition for Mo $(379.8 \mathrm{~nm})$ can both be accessed using the laser dye Exalite 376. The Mo transition is nearly outside the tuning range for this dye; however, the dye laser was able to produce sufficient intensity at this wavelength $(>0.1 \mathrm{~mJ} /$ pulse $)$ to saturate this particular Mo resonance transition anyway. For Re, Os, and Ir, spectroscopic data are less complete with many observed emission lines not assigned. However, a number of RIS schemes for Re and Os have been studied previously in our laboratory. ${ }^{11}$ Using data from Ref. 11, resonance transitions for these two elements were identified that could be accessed by frequency doubling the output from a dye laser operating with coumarin 480 . To identify an appropriate Ir transition, emission lines reported in the literature within the frequency range of this dye were scanned. ${ }^{16}$ A transition at $237.3 \mathrm{~nm}$ was found to give a useful Ir signal and was used for this study.

Samples consisted of metal grains from the ordinary chondrites, Leedey (L6), Marion, IA (L6), New Concord (L6), Sharps (H3), and Dhajala (H3-4) (meteorite types and metamorphic grades are given in parentheses). In all cases, the 150-300 $\mu \mathrm{m}$ grains consisted completely of kamacite, as determined by scanning electron microscopy/energy dispersive spectroscopy (SEM/EDS) and electron probe analyses. Some of the meteoritic samples were polished sections mounted directly on target stubs for loading into SARISA. Other samples were individual grains excised from sections of the meteorite by microcoring. The excised samples were potted in nonconductive epoxy before polishing and mounting on target stubs. All samples were mounted on metal target stubs using conductive epoxy and were carbon-coated to prevent charging. In order to quantify our results, SARISA was calibrated at the beginning and end of each day. Molybdenum was calibrated using a pure Mo foil. Os and Re were calibrated from a previously synthesized Os-Re-Ni alloy. ${ }^{8}$

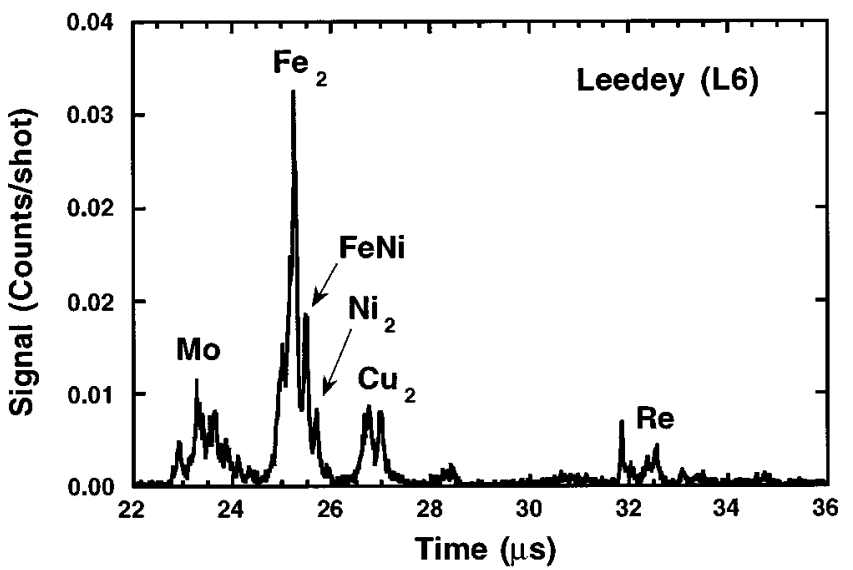

FIG. 3. Mass spectrum of a grain from the Leedey (L6) sample with dye lasers tuned on-resonance for Mo and Re. The mass spectrum is an average of 10000 laser shots.

Electron probe analyses determined the Os and Re concentrations in this alloy to be 824 and 957 parts per million atomic (ppma), respectively. The relative concentrations of $\mathrm{Ru}, \mathrm{Os}$, and Ir were calibrated from a natural laurite $\left(\mathrm{RuS}_{2}\right)$ crystal containing 6.2 at. \% Os and 2.9 at. \% Ir, as determined by SEM/EDS. Because the sputtering yield for laurite is unknown, sensitivity factors for $\mathrm{Ru}$ and Ir were obtained by comparing the Os signal in the laurite and Ni alloy standards.

Measurements were performed by tuning one dye laser to the resonance transition of either Mo or Ru and the other dye laser to the resonance transition of either Os, Re, or Ir. Mass spectra produced by a single ion pulse and laser shot were recorded by a transient digitizer. The spectra were internally averaged, and the averaged spectra were transferred to a personal computer for storage and analysis. The dye lasers were then tuned to another set of resonance transitions, and data were accumulated again. To separate the resonance signals from isobaric interferences, background spectra were collected by tuning both dye lasers away from the resonance frequencies. Typically, 20000 averages were collected for each determination. At the repetition rate of the experiments $(40 \mathrm{~Hz})$, this corresponds to $500 \mathrm{~s}$. Including data transfer time from the digitizer to the computer, each signal or background measurement required approximately 10 minutes.

\section{RESULTS}

Shown in Fig. 3 is a typical mass spectrum for Mo and Re in the Leedey (L6) sample obtained from 10000 laser pulses averaged together. The large peak near $25 \mu$ s is due to $\mathrm{Fe}_{2}$, $\mathrm{FeNi}$, and $\mathrm{Ni}_{2}$. Signals from atomic $\mathrm{Fe}$ and $\mathrm{Ni}$ (not shown in Fig. 3) are even larger but have been suppressed in order to protect the detector. The Mo and Re signals are near 23 and $32 \mu$ s, respectively, but are partially masked by isobars. To better see the signal, these two mass regions have been enlarged in Figs. 4 and 5. In Fig. 4 are shown mass spectra for resonantly ionized Mo and Ru from the Leedey (L6) sample, while Fig. 5 shows spectra for Re and Os from the Marion (L6) sample. Also shown in Figs. 4 and 5 are background 

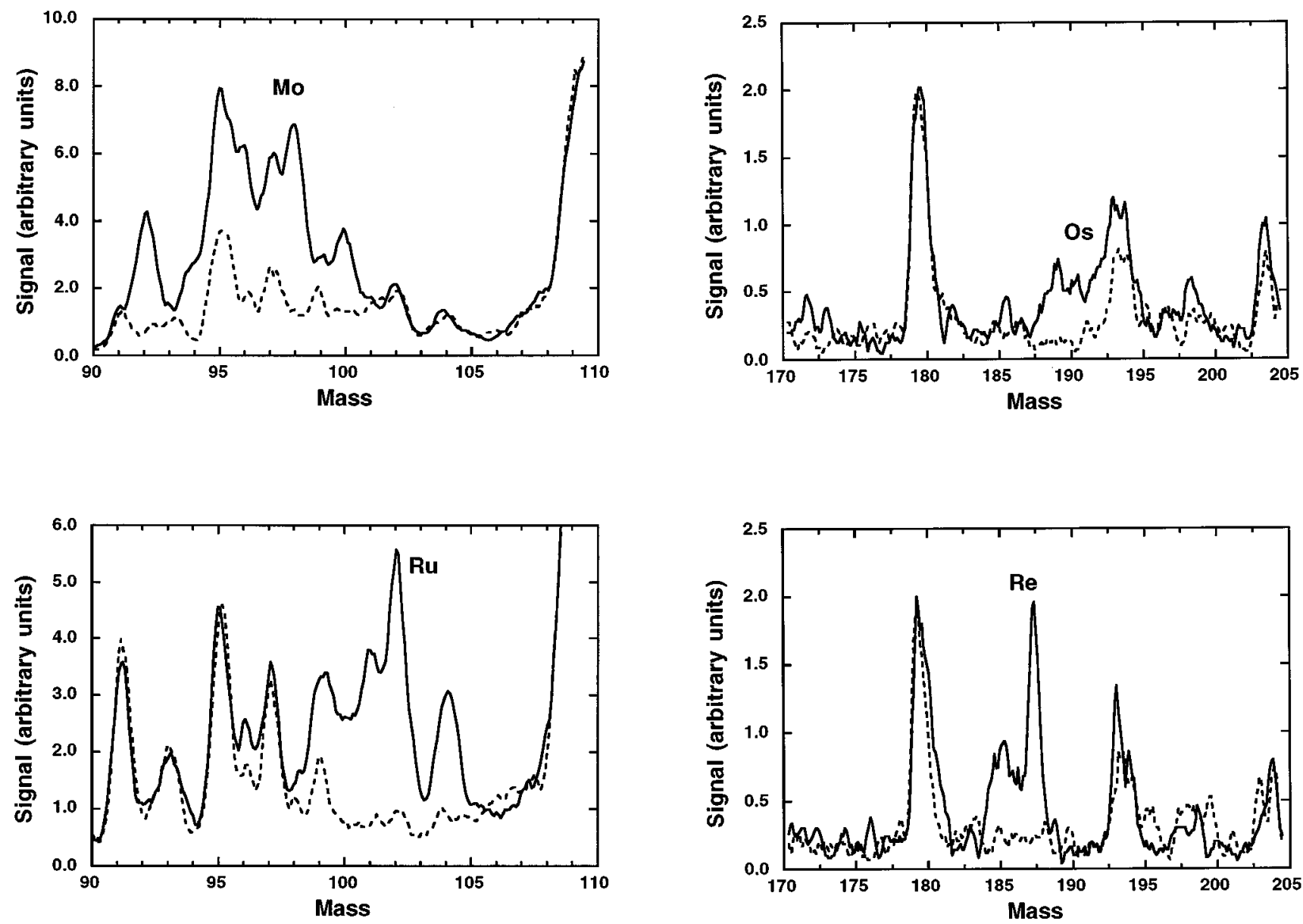

FIG. 4. Mass spectra of Mo and Ru from the Leedey (L6) sample. The measured concentrations are 4.7 and 1.4 ppma of $\mathrm{Mo}$ and $\mathrm{Ru}$, respectively. The solid lines are on-resonance for Mo (upper frame) or Ru (lower frame), while the dashed lines are off-resonance backgrounds. Each mass spectrum is an average of 15000 laser shots.

spectra obtained by tuning both dye lasers off their resonance frequencies. From these measurements, it was determined that the background was primarily due to nonresonance ionization independent of the dye laser wavelengths, and that a single background could be used for correcting all resonance signals.

Absolute concentrations of $\mathrm{Mo}, \mathrm{Ru}, \mathrm{Re}, \mathrm{Os}$, and Ir were determined by integrating spectra over the appropriate mass regions and taking the difference between the on- and offresonance results. Calibration standards having known concentrations of the five elements were analyzed at the beginning of each day to determine the useful yield (atoms detected per atom sputtered) of the instrument for each element. From the integrated signals, and by knowing the useful yield and the sputtering yields ${ }^{18}$ for the standard and meteoritic materials, absolute concentrations were determined. In total, eleven analyses were performed, each being the sum of 10000 to 20000 averages.

The primary purpose of this study was to document the feasibility and sensitivity of our multielement RIS analysis scheme for actual meteoritic samples. This has been accomplished and the results are presented in Table I. The concen-

FIG. 5. Mass spectra of Re and Os from the Marion (L6) sample. The measured concentrations are 0.10 and 0.77 ppma of Re and Os, respectively. The solid lines are on-resonance for Re (upper frame) or Os (lower frame), while the dashed lines are off-resonance backgrounds. Each mass spectrum is an average of 15000 laser shots.

trations of the five elements appear to vary considerably from one inclusion to another, even when the grains were from the same meteorite, although some of this variation may be due to the overlap of the sputter beam onto adjacent phases. Because insufficient attention in this test study has been paid to the reliability of our standards, instrumental drifts, sample charging, and ion beam positioning on grains, the accuracy of our present results are not particularly good, possibly being no better than a factor of two. Nevertheless, it

TABLE I. Ranges of concentrations and detection limits for Mo, Ru, Re, Os, Ir in metal inclusions from five meteorites. All concentrations are in ppm by atom. The detection limits were calculated assuming 20000 laser pulse averages.

\begin{tabular}{lcc}
\hline \hline & Detection limit & Concentration \\
\hline $\mathrm{Mo}$ & $0.055-0.42$ & $1-6$ \\
$\mathrm{Ru}$ & $0.018-0.11$ & $0.2-1.6$ \\
$\mathrm{Re}$ & $0.004-0.012$ & $0.03-0.16$ \\
$\mathrm{Os}$ & $0.036-0.19$ & $<0.06-1.2$ \\
$\mathrm{Ir}$ & $0.030-0.22$ & $<0.07-0.4$ \\
\hline \hline
\end{tabular}


is clear that, with additional work, accuracies can be obtained which are only limited by the counting statistics precisions.

We have calculated detection limits assuming that a statistically significant signal must be three times the standard deviation of the background-corrected measurement. Background levels varied considerably among grains; consequently detection limits have been calculated for each measurement. The range of detection limits for the five elements is also given in Table I, assuming a measurement consists of 20000 laser pulses. The variation in background levels from sample to sample is associated with difference in the magnitude of isobaric molecular signals that interfere with detection of the elements of interest. Whether these interferences are due to differences in composition of the grains being measured or difference in overlap of the ion beam with adjacent phases is unclear. Nevertheless, from Table I it is observed that the measured concentrations of these elements in ordinary chondritic metals is usually substantially above the determined detection limits, demonstrating the sensitivity of our analytical technique for this application.

\section{DISCUSSION}

\section{A. In situ analyses of meteorites}

To assess the precision of the measurements, two separate determinations for the Leedey sample were performed on the same day. Both measurements were performed on the same spot; however, the first measurement was near the polished surface and the second was taken after ion milling away material to a depth of approximately $30 \mu \mathrm{m}$. Assuming uniform distributions of trace elements, differences between the two measurements are an indication of the precision. This test suggests that the precision of the present data is about $30 \%$. A second means of assessing the precision is to examine results from the daily calibration measurements. Measurements on Mo in a pure Mo foil and $\mathrm{Ru}$, Os, and $\mathrm{Ir}$ in laurite were performed at the beginning and end of each day. Comparison of these results for six days showed that over the period of a day, the useful yield changed about $20 \%$ on average, typically decreasing with time.

In these measurements the precision was limited by a combination of changes in (1) ionization efficiency due to degradation of laser power, (2) the overlap between the laser ionization volume and extraction volume of the mass spectrometer, (3) ion beam current striking the grain due to loss of beam alignment, and (4) the transmission of the mass spectrometer. In future measurements it is anticipated that better regulation of instrumental conditions will easily result in significant improvements to the precision.

\section{B. Analysis time improvements}

As noted above, one aim of this research is to develop methods that reduce analysis times while retaining the high sensitivity and low sample consumption rate that are inherent with RIS measurements. We have demonstrated for the first time that RIS analyses can be made in parallel by measuring two elements simultaneously, and thus have reduced by a factor of two the time required for the analysis and the amount of sample consumed per element analyzed. Most significantly, the accuracy of element ratios measurements (more important than absolute concentrations for meteoritic problems) is significantly improved. There is some added complexity to the laser system for these measurements, mostly in setting up an additional ionization scheme and in assuring proper time and spatial overlap, but it appears that the precision and accuracy of the analyses are not adversely affected by the multielement measurements.

From our dual-element measurements, it is apparent that extension of this technique to multielement parallel RIS/ SNMS analyses is possible. Further, there should be no limit to the number of elements that could be analyzed simultaneously by this method. All that is needed is a parallel detection system for all masses (e.g., a TOF mass spectrometer) and a sufficient number of lasers to produce the required wavelengths of light. Clearly, extrapolating parallel RIS analyses to the limit of all elements is impractical from the standpoint of the number of lasers required, but also because there are isobaric interferences between elements, as is the case for $\mathrm{Mo} / \mathrm{Ru}$ and $\mathrm{Re} / \mathrm{Os} / \mathrm{Ir}$.

By choosing ionization schemes for these measurements that match the three criteria outlined above, additional benefits beyond the simplicity of the laser system were achieved. To understand this, consider how background subtractions in RIS measurements work. One of the substantial advantages of RIS versus other postionization methods is its ability to differentiate between signal and background easily. As described above, this is accomplished by changing the frequency of the dye laser on and off the resonant transition of the element of interest. The background detected in this fashion has three components: (1) electronic noise generated within the detector system; (2) stray ions reaching the detector, such as secondary ions; and (3) photoions that are not generated by RIS. The contributions of the three components to the background can be assessed by measuring backgrounds with the laser and ion beam on and off. What is typical, as well as what was observed in these measurements, is that photoions produced by multiphoton ionization processes generally comprise the majority of the background. Because of this, ionizing laser intensities used in trace analysis measurements are always carefully controlled. Intensities are set high enough to saturate transitions, but no higher, so that two-photon processes (that are proportional to the laser intensity squared) are minimized.

It is generally the case that the laser intensity required to saturate a resonant transition is orders of magnitude smaller than the intensity required to saturate the ionization step. Thus, in a typical measurement, the majority of the photoiongenerated background is caused by the laser used for the ionization step. In the case of the two-color, two-photon ionization schemes that we employed, photons from the excimer laser were used for the ionization step for all five elements. This explains why the background for these measurements was found to be nearly independent of the wavelength of the dye lasers. Thus, through the choice of ionization schemes, it was possible to reduce the number of background measurements, since one background measurement suffices for five elements. The savings in analysis time and sample consump- 
tion associated with collecting a single background spectrum is substantial. When combined with the savings associated with simultaneous dual-element analyses, only about a third of the usual amount of instrument time is required. Therefore, determining the concentration of five elements in an inclusion required about thirty minutes instead of the previously required one and one-half hours.

The time that has been saved in these measurements has come at a cost of reduced selectivity and sensitivity that is a consequence of the ionization schemes that were used. Our RIS/SNMS measurements of Mo, Os, and Ir in metallic grains are all adversely affected by molecular ion interferences. A consequence of this is that detection limits for Mo in Table I are three-to-five times higher than those for Ru. As can be seen in Fig. 4, this is a result of nonresonantly ionized molecules that overlap the Mo signal. Peaks in the background spectra at masses 91, 93, 95, 97, and 99 are believed to be nonresonantly ionized $\mathrm{FeCl}, \mathrm{NiCl}, \mathrm{KFe}$, and $\mathrm{KNi}$. The three latter molecules interfere with detection of Mo. On the other hand, $\mathrm{Ru}$ is at higher mass where little interference occurs (Fig. 4). The same is true for Re, Os, and Ir, as seen in Fig. 5. There are two unidentified peaks in the background spectra at masses of 179 and 193. The signal for Re falls between these molecules, but both Os and Ir are overlapped by the peak at 193. This is reflected in the detection limits for these elements, where the value for Re is nearly an order of magnitude better than that for Os or Ir. In extreme cases, detecting a small concentration of one element in the presence of a large concentration of another species of similar mass may not be possible using the simple two-color schemes that were employed in this study. An example might be indium. As can be seen in Fig. 3, because the samples have substantial $\mathrm{Ni}$, the ${ }^{58} \mathrm{Ni}_{2}$ nonresonant signal is very large, and it would be difficult to detect ${ }^{116}$ In at low concentrations with any ionization scheme that uses $308 \mathrm{~nm}$ photons.

There is a trade-off between the detection limit and the ionization scheme that needs to be considered before attempting similar simultaneous analyses. Clearly, the added complexity of a three-color scheme is needed when extremely low concentrations must be determined (below 1 $\mathrm{ppb}),{ }^{19}$ or when a particular element is masked by an isobar. $^{5,7,8,11}$ However, many analyses do not stress instrumental detection limits to such extremes, and so simpler ionization schemes suffice. In such situations, the simpler ionization schemes allow more flexibility in choosing resonance transitions and reduce the demands on laser equipment, so that multielement analyses may be accomplished quicker and easier. It appears that in situ determinations of Mo, Ru, Re, Os, and Ir in metal grains from meteorites is such an example.

\section{CONCLUSIONS}

Simultaneous dual-element RIS/SNMS measurements have been performed for the first time. It was shown that Mo or $\mathrm{Ru}$ could be detected coincident with $\mathrm{Re}$, Os, or Re and that by carefully selecting two-color, two-photon ionization schemes, the concentration of all five elements could be determined in about one-third the time of more traditional RIS/
SNMS measurements. The measurement technique was used for in situ determinations of Mo, Ru, Re, Os, and Ir in ordinary chondrite grains. A consequence of the ionization schemes that were chosen was that nonresonant photoionization of isobars dominated the background, producing higher detection limits than are achievable with three-color schemes. However, it is believed that the benefits derived from performing parallel RIS measurements outweighs the reduced detection limits for most applications.

\section{ACKNOWLEDGMENTS}

The authors thank Paul Carpenter and John Armstrong for their assistance with the SEM and electron probe analyses. Joel Blum is thanked for providing the Ni-Os-Re standard and George Rossman for the laurite sample. This work was supported by the U.S. Department of Energy, BES-Materials Sciences, under Contract No. W-31-109-ENG-38 (ANL) and by NASA Grant No. NAGW 3592 (Caltech).

\footnotetext{
${ }^{1}$ M. J. Pellin, C. E. Young, W. F. Calaway, J. E. Whitten, D. M. Gruen, J. D. Blum, I. D. Hutcheon, and G. J. Wasserburg, Philos. Trans. R. Soc. London A 333, 133 (1990).

${ }^{2}$ S. W. Downey and R. S. Hozack, J. Vac. Sci. Technol. A 8, 791 (1990). ${ }^{3}$ N. Thonnard, H. F. Arlinghaus, R. D. Willis, E. H. Taylor, M. C. Wright, W. A. Davis, M. T. Spaar, and L. J. Moore, in Resonance Ionization Spectroscopy and Its Applications RIS90, edited by J. E. Parks and N. Ometto, Inst. Phys. Conf. Series No. 114 (The Institute of Physics, Bristol, 1990), pp. 271-274.

${ }^{4}$ D. L. Pappas, D. M. Hrubowchak, M. H. Ervin, and N. Winograd, Science 243, 64 (1989).

${ }^{5}$ D. R. Spiegel, W. F. Calaway, G. A. Curlee, A. M. Davis, R. S. Lewis, M. J. Pellin, D. M. Gruen, and R. N. Clayton, Anal. Chem. 66, 2647 (1994). ${ }^{6}$ W. F. Calaway et al., in Resonance Ionization Spectroscopy and its Applications RIS92, edited by C. M. Miller and J. E. Parks, Inst. Phys. Conf. Series No. 128 (The Institute of Physics, Bristol, 1992), pp. 271-274.

${ }^{7}$ D. R. Spiegel et al., Anal. Chem. 64, 469 (1992).

${ }^{8}$ J. D. Blum, M. J. Pellin, W. F. Calaway, C. E. Young, D. M. Gruen, I. D. Hutcheon, and G. J. Wasserburg, Geochim. Cosmochim. Acta 54, 875 (1990).

${ }^{9}$ H. Oechsner, Anal. Chim. Acta 283, 131 (1993).

${ }^{10}$ C. H. Becker, S. G. MacKay, and D. G. Welkie, J. Vac. Sci. Technol. B 10, 380 (1992).

${ }^{11}$ J. D. Blum, M. J. Pellin, W. F. Calaway, C. E. Young, and D. M. Gruen, Anal. Chem. 62, 209 (1990).

${ }^{12}$ K. P. Jochem, M. Seufert, and F. Begemann, Z. Naturforsch. 35a, 57 (1980).

${ }^{13}$ E. R. Rambaldi, H. Wanke, and J. W. Larimer, in Proceedings of the 10th Lunar Planetary Science Conference, Geochim. Cosmochim. Acta Suppl. II (Pergamon, New York, 1979), pp. 997-1010.

${ }^{14}$ B. Fegley and H. Palme, Earth Planet. Sci. Lett. 72, 311 (1985).

${ }^{15}$ D. L. Lindstrom, Nucl. Instrum. Methods A 299, 584 (1990).

${ }^{16}$ C. E. Moore, Atomic Energy Levels (U.S. GPO, Washington, DC, 1971), Vol. III.

${ }^{17}$ S. R. Coon, W. F. Calaway, M. J. Pellin, J. W. Burnett, and J. M. White, Surf. Interface Anal. 20, 1007 (1993).

${ }^{18}$ N. Matsunami et al., At. Data Nucl. Data Tables 31, 1 (1984)

${ }^{19}$ D. M. Gruen, W. F. Calaway, M. J. Pellin, C. E. Young, D. R. Spiegel, R. N. Clayton, A. M. Davis, and J. D. Blum, Nucl. Instrum. Methods B 58, 505 (1991).
} 